\title{
p120-catenin isoform 3 regulates subcellular localization of Kaiso and promotes invasion in lung cancer cells via a phosphorylation-dependent mechanism
}

\author{
PENG-XIN ZHANG, YAN WANG, YANG LIU, GUI-YANG JIANG, QING-CHANG LI and EN-HUA WANG \\ Department of Pathology, First Affiliated Hospital and College of Basic Medical \\ Sciences of China Medical University, Shenyang 110001, P.R. China
}

Received January 24, 2011; Accepted March 18, 2011

DOI: $10.3892 /$ ijo.2011.995

\begin{abstract}
E-cadherin stability at the plasma membrane as well as Rho GTPase activity in the cytoplasm, and also interacts with the transcriptional repressor, Kaiso, in the nucleus. However, the role of different isoforms and the phosphorylated state of p120-catenin in the nucleus is poorly understood. In the present study, we show that p120catenin isoform 3 interacts with Kaiso in lung cancer cells by immunoprecipitation. Nuclear-cytoplasmic extraction and immunofluoresence confirmed that Kaiso shuttled out of the nucleus via p120-catenin isoform 3 . The cytoplasmic enrichment of Kaiso by p120-catenin isoform 3 was abolished due to the inhibition of chromosomal region maintenance-dependent nuclear export via leptomycin. The lung tumor tissue and cell lines expressed higher levels of the serine 288 phosphorylated form. Also, serine 288 phosphorylation in p120-catenin isoform 3 enhanced the binding with Kaiso. Moreover, immunofluoresence and transwell invasion assay showed that the phosphorylation of serine and threonine sites in p120-catenin induced F-actin remodelling and promoted the invasion of lung cancer cells. Collectively, our data establish that p120catenin isoform 3 regulates the nuclear export of Kaiso and promotes invasion in lung cancer cells via a phosphorylationdependent mechanism. Serine 288 phosphorylation can contribute to lung cancer progression.
\end{abstract}

\section{Introduction}

The Armadillo catenin ( $\beta$-catenin, $\gamma$-catenin and p120-catenin) regulates E-cadherin activity through different mechanisms $(1,2)$. $\beta$-catenin and $\gamma$-catenin compete for binding to the

Correspondence to: Professor En-Hua Wang, Department of Pathology, China Medical University, 92 2nd North Rode, Heping District, Shenyang 110001, P.R. China

E-mail: wangeh@hotmail.com

Key words: p120-catenin, Kaiso, phosphorylation, invasion
E-cadherin catenin binding domain. However, unlike the classical catenins ( $\beta$ - and $\gamma$-), p120-catenin (hereafter p120ctn) binds to E-cadherin at a distinct conserved juxtamembrane domain to regulate E-cadherin stability at the plasma membrane (3-5). When E-cadherin is downregulated or lost, this results in the redistribution of p120ctn to the cytoplasm and in the interaction with Rho GTPase to regulate cytoskeleton organization (6-9). Increasing evidence has revealed that p120ctn also shuttles into the nucleus to regulate gene expression via interaction with transcription factor Kaiso (10). Unlike the classical catenins, p120ctn is also expressed as multiple isoforms owing to four distinct ATG start codons at the amino terminus and exons A, B, C at the carboxy terminus (11). There is growing interest in the biological function of these isoforms. Certain researchers have found that sequences within the p120ctn N-terminus (1-347 amino acids) are prerequisites for both nuclear localization and the p120ctn-induced branching phenotype. The exon B-encoded sequences are prerequisites for exclusion from the nucleus (12). Other data have indicated that the increased expression of p120 isoform 1 during tumor progression contributes to the invasive phenotype. The larger p120ctn type 1 isoforms do not interact with Kaiso, but the isoform 3 that has the 102-end amino acids of the p120ctn isoform 1, can interact with Kaiso $(10,13)$.

Kaiso is a member of the Broad complex, Tramtrak, Bric à brac/Pox virus and zinc finger (BTB/POZ) subfamily of zinc finger proteins, that was originally identified in a yeast-twohybrid screen for binding partners of p120ctn (10). However, unlike any of the previously characterized POZ proteins, Kaiso exhibits dual-specificity DNA binding: Kaiso recognizes a sequence-specific DNA consensus, TCCTGCnA, as well as methylated CpG-dinucleotides $(14,15)$. A subset of Kaiso target genes include the $\beta$-catenin/TCF targets, cyclin Dl and matrilysin (MMP-7). Other target genes identified to date include MTA2, Wnt-11, siamois and rapsyn (16-18). The overlap of the p120ctn binding site with the Kaiso DNA-binding ZF domain allows for the nuclear p120ctn to regulate Kaiso DNA-binding and ultimately transcriptional activity.

Several investigators have discovered p120ctn entries and shuttles out of the nucleus via nuclear localization signal (NLS) and nuclear export signal (NES). Leptomycin B (LMB) can inhibit chromosomal region maintenance (CRM-1) receptor- 
mediated nuclear export that causes nuclear accumulation of proteins containing NES $(19,20)$. However, Kaiso has NLS (21), its NES remains unexplored, but its nuclear or cytoplasmic distribution couples with p120ctn $(22,23)$. Moreover, p120ctn phosphorylation acts as a mechanism in the regulation of E-cadherin stability and Rho GTPase activity $(24,25)$, but little research has been done on the phosphorylated p120ctn in the nucleus. Serine 288 phosphorylation in p120ctn is a likely event in the nucleus (26), we postulated that Kaiso shuttles out of the nucleus via NES in p120ctn and that serine 288 phosphorylation affects p120ctn interaction with Kaiso.

In this study, we transfected p120ctn plasmids into lung cancer cell lines, used LMB to inhibit p120ctn shuttling out of the nucleus, and the PKC pathway activator, PMA, and its inhibitor, BisI, to alter the phosphorylated state of p120ctn. We then observed nuclear-cytoplasmic distribution of p120ctn and Kaiso. Furthermore, we investigated the role of nuclear p120ctn and its phosphorylated state on binding with Kaiso and cell invasion.

\section{Materials and methods}

Cell culture and treatment. The BE1 and LH7 cell lines were established from a human pulmonary giant cell carcinoma (a gift from Dr J. Chen, Medical College of Beijing University, China). The human pulmonary carcinoma cell lines, NCI-H460, NCI-661, LTEP-a-2, SPC-A-1, SK-MES-1 and NCI-H446, were obtained from the Cell Bank of Chinese Academy (Shanghai, China). Immortal bronchial epithelial HBE and adenocarcinoma A549 cell lines were obtained from the American Type Culture Collection (Manassas, VA, USA). The cell lines were maintained in DMEM (A549) and RPMI1640 medium (the other cell lines) (Gibco Inc., Los Angeles, CA, USA), supplemented with 10\% FBS (Gibco). For serum starvation experiments, cells were starved in DMEM containing $0.1 \%$ FBS. Leptomycin (Sigma-Aldrich Inc., St. Louis, MO, USA, $50 \mathrm{nM}$ ), PMA (Sigma, $200 \mathrm{nM}$ ) or BisI (Sigma, $1 \mu \mathrm{M}$ ) were added to the cell medium.

Plasmid constructs and transfection. Full-length murine p120ctn isoform $1 \mathrm{~A}$ and $3 \mathrm{~A}$ were generated by PCR using RacCMV-Kpnl/p120-1/3A (a gift from A.B. Reynolds, Vanderbilt University Medical School, Nashville, TN, USA) as the template, then the PCR products were subcloned into the nucleus-localization expression vector $\mathrm{pCMV/myc/nuc}$ (Invitrogen, Carlsbad, CA, USA) to generate the $\mathrm{pCMV} /$ p120ctn-1A/nuc (p120-NLS-1A) and pCMV/p120ctn-3A/nuc (p120-NLS-3A) plamids. To generate p120ctn deletion mutants, the fragment of p120-catenin from amino acids 46, 102, 350 to 933 was obtained by PCR using RacCMV-Kpnl/ p120-1 as the template. The PCR products were digested and overhang ends were filled with Klenow polymerase, and were then ligated into cDNA3.1 (Invitrogen) to construct the mutants. Serine 288 site mutation was obtained using the Quickchange XL Site-Directed Mutagenesis Kit (Stratagene, Cedar Creek, TX, USA) according to the manufacturer's instructions, using RacCMV-Kpnl/p120-1/3A as the template to generate P120-1A and 3A S288E/S288A point mutant plasmids. The pBluescript-Kaiso plasmid was a gift from Juliet M. Daniel (McMaster University, Hamilton, ON, Canada). Human specific p120ctn siRNA sequences A:5'-GGATCACAGTCACC TTCTA-3', 5'- TAGAAGGTGACTGTGATCC-3'; B: 5'-GCA CTTGTATTACAGACAA-3', 5'-TTGTCTGTAATACA AGTGC-3' C: 5'-GGATAACAAGATTGCCATA-3', 5'-TATGGCAATCTTGTTATCC-3', were synthesized by Takara (Takara Biotechnology Co., Ltd., Dalian, China). The DNA plasmids were transfected with Lipofectamine ${ }^{\mathrm{TM}} 2000$ (Invitrogen) according to the manufacturer's instructions.

Tissue sample and immunohistochemitry staining. Tumor specimens from 50 patients with NSCLC were obtained following surgical resection at the First Affiliated Hospital of China Medical University. Twenty cases (included in the 50 cases) of tumor and paired non-tumor portion (with $>5 \mathrm{~cm}$ distance from the primary tumor edge) of the same case were quickly frozen and maintained at $-70^{\circ} \mathrm{C}$ for protein analysis. All specimens were evaluated for diagnosis following the criteria for classification of lung cancer by the World Health Organization (27), and 38 squamous cell carcinomas and 22 adenocarcinomas were confirmed. This study was conducted with the approval of the Institutional Review Board of China Medical University. Immunohistochemistry was performed using established protocols (9) with monoclonal anti-p120ctn and anti-Ps288 (1:400 dilution, BD Biosciences, Bedford, MA, USA) antibodies.

Immunofluorescence. Twenty-four hours post-transfection, cells were fixed (4\% paraformaldehyde), permeabilized $(0.2 \%$ Triton-X-100) and blocked (5\% BSA) before being incubated with anti-p120ctn and anti-Ps288 $\mathrm{mAb}(1.25 \mathrm{mg} / \mathrm{ml}, \mathrm{BD}$ Biosciences), anti-Kaiso pAb (2 mg/ml, Santa Cruz Biotechnology, Santa Cruz, CA, USA) and TRITC-phalloidin F-actin (1:1000, Invitrogen) antibodies. This was followed by incubation with a second antibody conjugated to TRITC/FITC-labeled (1:200, Zhongshan Golden Bridge Biotechnology Co., Beijing, China). Bisbenzimide (Hoechst 33342, Sigma) was used for nuclear counterstainings.

Nuclear and cytoplasmic extraction, immunoprecipitation and immunoblot analysis. Nuclear and cytoplasmic extraction (Pierce Biotechnology, Inc., USA) was performed according to the manufacturer's instructions. Immunoprecipitation and immunoblotting in frozen tissue and cell lines were carried out using established protocols $(9,22)$ with anti-p120ctn pp120, 6H1 (Santa Cruz), anti-Ps288, anti-Kaiso (Upstate Biotechnology, Lake Placid, NY, USA), anti-lamin B1 (Santa Cruz), antitubulin (Santa Cruz), anti-actin (Upstate) $\mathrm{mAb}$ and anti-Kaiso $\mathrm{pAb}$, at a dilution of 1:500-800. Bound antibody was detected by incubation with HRP conjugated secondary antibody (1:5,000-1:10,000, Zhongshan Golden Bridge) and processed using enhanced chemiluminescence. When necessary, blots were stripped and reprobed with additional antibodies.

Matrigel invasion assay. Matrigel (BD Biosciences) and transwell inserts of $8 \mu \mathrm{m}$ pore size (Corning Inc., Corning, NY, USA) were used to assess the invasive ability of cells. The upper chamber was seeded with $5 \times 10^{5}$ cells $/ \mathrm{ml}$ and allowed to migrate at $37^{\circ} \mathrm{C}$ for $48 \mathrm{~h}$ towards a lower reservoir of DMEM with $10 \%$ FBS before fixing, staining and counting cells on the lower surface filters. 


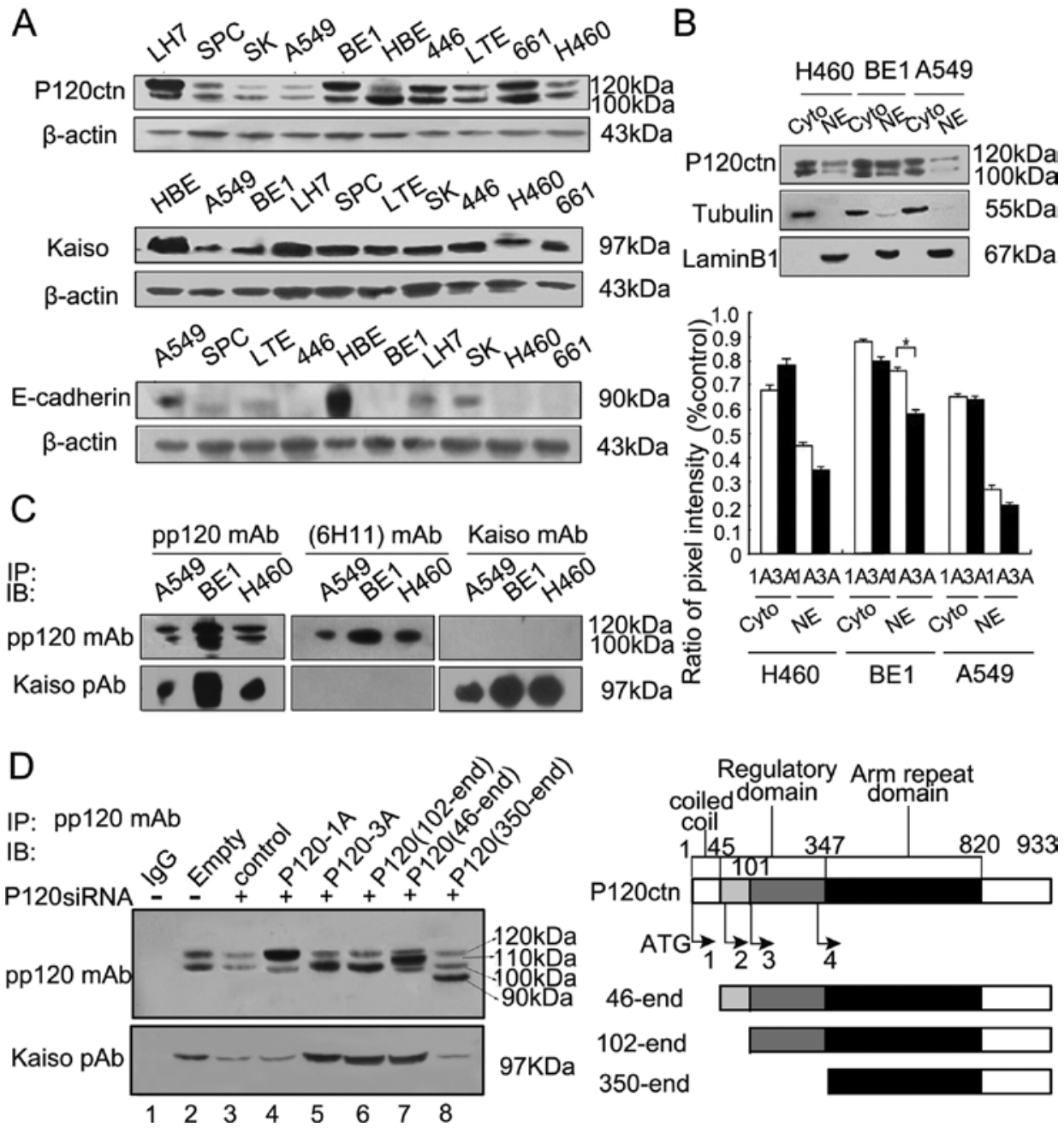

Figure 1.p120ctn isoform 3 interacts with Kaiso. (A) p120ctn, Kaiso and E-cadherin were analysed by Western bloting with anti-p120, anti-Kaiso and anti-Ecadherin mAb in lung cancer cell lines. (B) A549, BE1 and H460 cells were analysed by nuclear-cytoplasmic extraction and Western blotting to assess the relative levels of p120ctn within each compartment. The efficiency of cytoplasmic (Cyto) and nuclear (NE) fraction was assessed by tubulin and lamin B1, respectively. For the quatification of band density, the fold elevation of p120ctn isoform in the cytoplasm versus the nucleus was displayed as the mean \pm SD ${ }^{*} \mathrm{P}<0.05$. (C) p120ctn and Kaiso were immunoprecipitated by p120ctn mAb pp120, $6 \mathrm{H} 11$ (recognized only p120ctn isoform 1) and Kaiso mAb as bait, then analysed by Western blotting with pp120 mAb and Kaiso pAb. (D) The vector alone (Empty) was transfected into A549 cells and the mutant plasmids into p120RNAi A549 cell lines. The protein was extracted and immunoprecipitated by pp120 mAb, then Western blotted with Kaiso pAb (left panel). Diagram of the different domains of p120-catenin. Alternative splicing in the N-terminal domain gives rise to isoforms 1,2,3 and 4, each initiating at distinct ATG start codons (1, 55, 102 and 340, respectively). The regulatory domain capable of being phosphorylated in vitro. The arm repeat domain is Kaiso binding sites. The length and composition of the mutant plasmid fragments used (46,102,350 to end) in this study are shown (right panel).

Statistical analysis. All data were expressed as the means \pm standard deviation (SD) for in vitro experiments, performed at least three times, and data were analysed using one-way analysis of variance using SPSS 13.0 for Windows (SPSS Inc., Chicago, IL, USA). P-values of $<0.05$ were considered to be statistically significant.

\section{Results}

p120ctn isoform 3 interacts with Kaiso. We assessed p120ctn protein expression in various lung cancer cell lines. Cells predominantly expressed the p120ctn isoform $1(120 \mathrm{kDa})$ and isoform $3(100 \mathrm{kDa})$. We selected A549 and H460 cell lines, which the latter showed E-cadherin loss and both showed low expression of p120ctn and Kaiso (Fig. 1A). As shown in Fig. 1B, nuclear-cytoplasmic extraction analysis was performed to measure the cellular distribution of p120ctn isoforms. p120ctn isoform 1 and 3 were both localized in the nucleus and cytoplasm in the A549, BE1 and H460 cells. Moreover, nuclear p120ctn isoform 1 expression was higher than isoform 3 expression in $\mathrm{BE} 1$ cell lines (Fig. $1 \mathrm{~B}, \mathrm{P}<0.05)$. To determine the effect of p120ctn isoforms on Kaiso binding affinity Fig. 1C), an immunoprecipitation assay was carried out which showed that Kaiso co-precipitated efficiently with total p120ctn isoforms antibody, pp120, but not with isoform 1 specific antibody, $6 \mathrm{H} 11$. This observation demonstrates that Kaiso can interact only with the smaller p120ctn isoform 3.

To study the difference in binding of p120-1A and p120-3A to Kaiso, we stably transfected several p120ctn deletion mutants into A549 cell lines, in which p120ctn was knocked down by siRNA. As shown in Fig. 1D, the immunoprecipitation assays demonstrated that the overexpressed p120ctn form comprising of amino acids 46 to the end, which lacked coiled coil domain, co-precipitated more with Kaiso than the control 


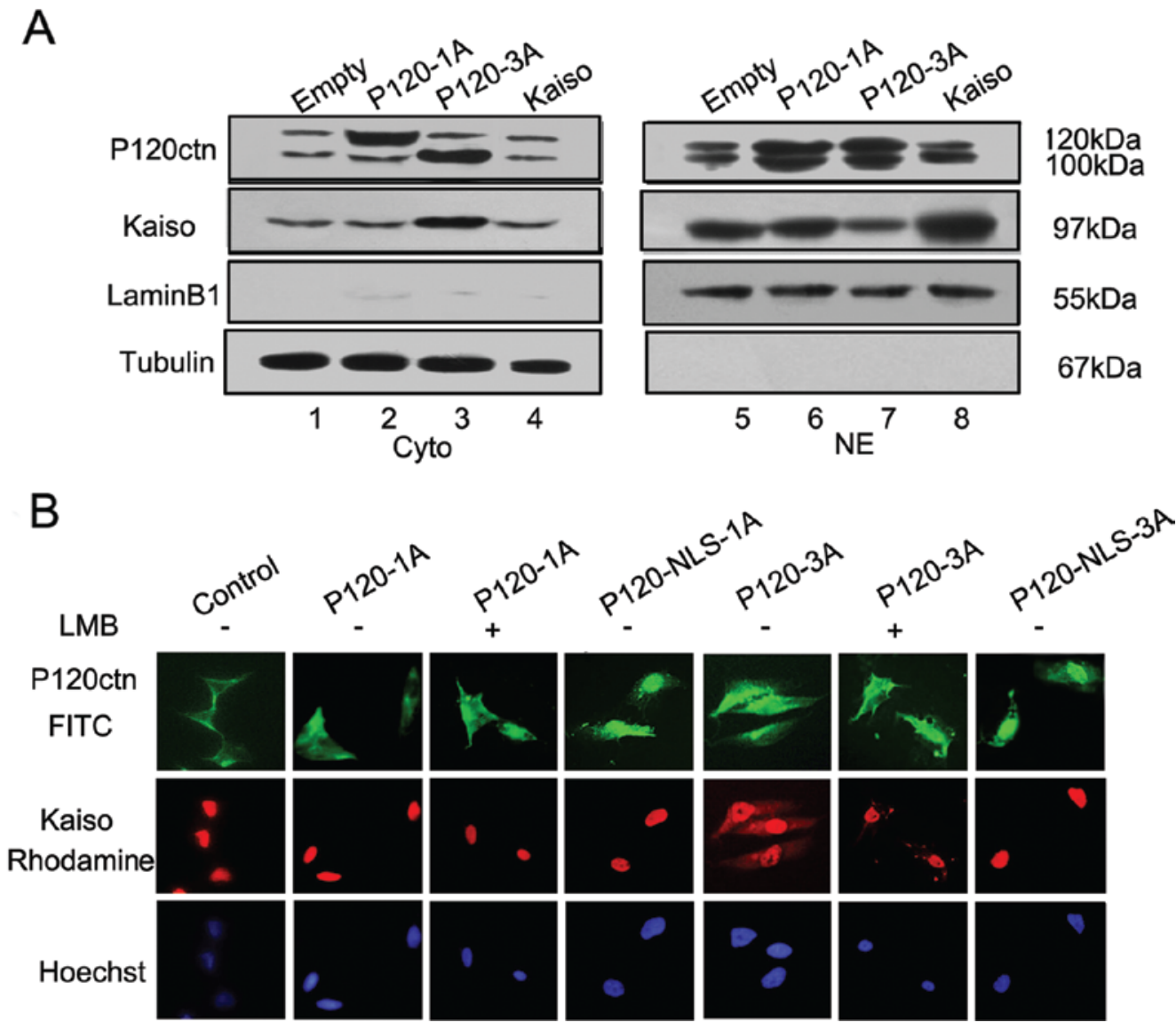

Figure 2. p120ctn isoform 3 assists the nuclear export of Kaiso. (A) Nuclear-cytoplasmic extraction and Western blotting were carried out in A549 and A549 cells overpressing p120-1A/3A and Kaiso plasmids. (B) In A549 cells overexpressing p120-1A/3A and p120-NLS-1A/3A, LMB (+) was added. They were then double-stained with p120ctn and Kaiso antibodies to visualize p120ctn (FITC-labelled, green) and Kaiso (TRITC-labelled, red) distribution. Nuclei were visualized with Hoechst dye (blue).

(compare lane 3 to lane 7). However, the Kaiso association with the sequence from amino acid 350 to the end, which lacked the regulatory domain of p120ctn, was similar to that observed in the control (compare lane 3 to lane 8). Kaiso interacted similarly with the p120ctn fragment comprising of amino acid 102 to the end and the overexpressed p120-3A (compare lane 5 to lane 6). Therefore, these results suggest that 1-350 amino acid residues in p120ctn affect the binding with Kaiso, although the arm repeat domain in p120ctn is a prerequisite for Kaiso binding.

P120ctn isoform 3 assists the nuclear export of Kaiso and is largely CRM-dependent. We postulated that p120ctn assisted Kaiso in shuttling out of the nucleus via its NES through the CRM-1 pathway. To test our hypothesis, we transiently transfected p120-1A, p120-3A and Kaiso plasmids into A549 cells. As shown in Fig. 2A, nuclear-cytoplasmic extraction showed that cytoplasmic Kaiso was increased (Fig. 2A compare lane 1 to lane 3) and nuclear Kaiso was decreased (compare lane 5 to lane 7) by p120-3A overexpression as opposed to vector alone overexpression. However, the amount of nuclear and cytoplasmic Kaiso protein remained unchanged by p120-1A overexpression compared to vector alone overexpression (compare lane 5 to lane 6 and lane 1 to lane 2). As shown in Fig. 2B, immunofluorescence also showed that cytoplasmic p120ctn staining was elevated. Cytoplasmic Kaiso staining was also elevated by p120-3A overexpression (Fig. 2B). These data suggest that the nuclear export of Kaiso could be mediated by p120ctn isoform 3 . We then investigated the influence of the CRM-1 inhibitor, leptomycin B, on the ability of nuclear enrichment of p120ctn in A549 cells. Significant nuclear enrichment of p120ctn occurred within $4 \mathrm{~h}$, and peaked by $18 \mathrm{~h}$ (Fig. 3A). As shown in Fig. 3B, nuclear-cytoplasmic extraction analysis showed that nuclear p120ctn was partially increased due to p120-3A overexpression and LMB treatment (Fig. 3B, compare lane 10 to lane 12). Kaiso showed a subsellular redistribution of nuclear increase and cytoplasmic decrease compared to only p120-3A overexpression (compare lane 10 to lane 12 and lane 3 to lane 5). This result indicates that the Kaiso enrichment in the cytoplasm by p120ctn 3 is largely CRM-1-dependent. However, nuclear Kaiso was increased and cytoplasmic Kaiso was unchanged by Kaiso overexpression (Fig. 2A, compare lane 5 to lane 8 and lane 1 to lane 4). When p120-NLS-3A was overexpressed, p120ctn predominantly accumulated in the nucleus (Fig. 3B, compare lane 12 to lane 14), and no subcellular redistribution of Kaiso occurred compared to vector alone transfection (Fig. 3B, compare lane 1 to lane 7 and lane 8 to lane 14), demonstrating that Kaiso entering into the nucleus is independent of p120ctn isoform 3. However, the subcellular distribution of 
A
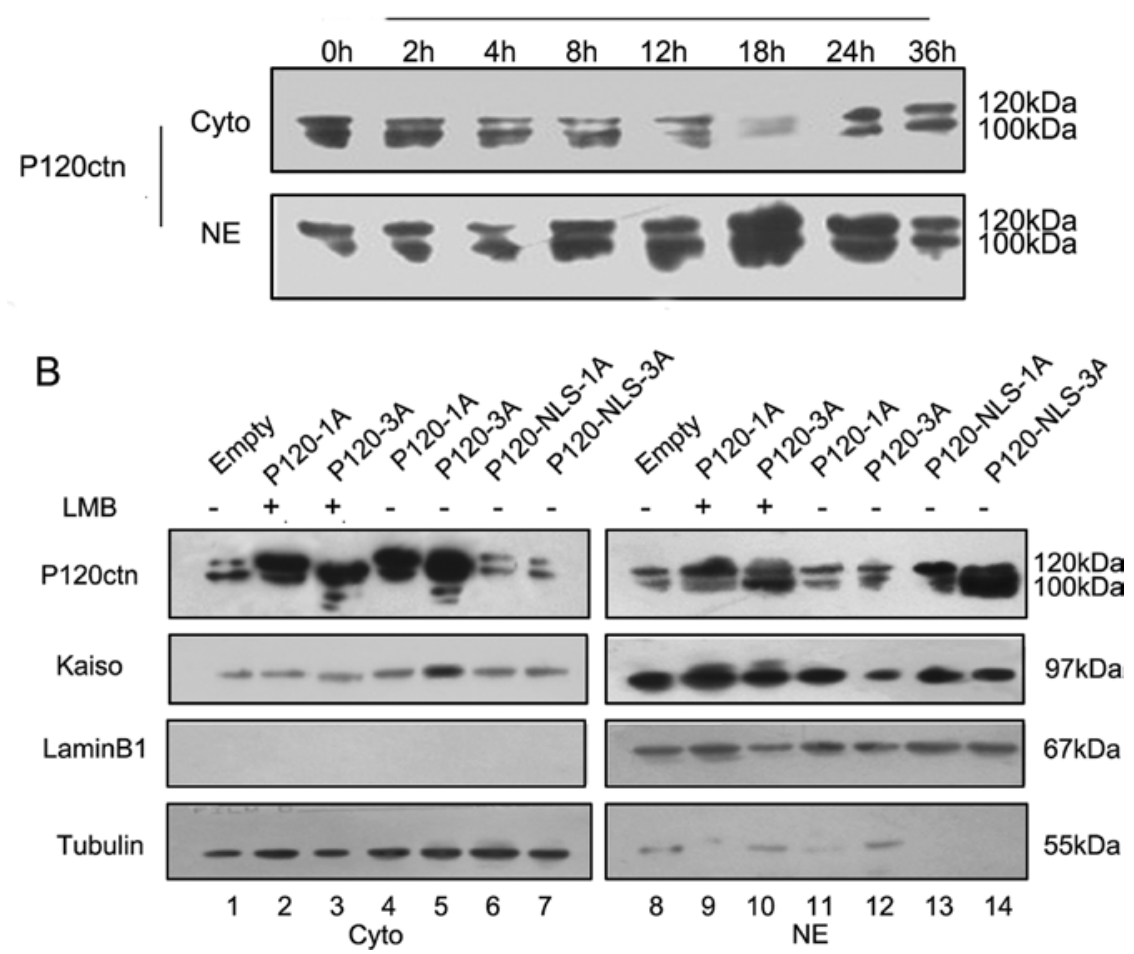

Figure 3. p120ctn isoform 3 assists the nuclear export of Kaiso and is largely CRM-dependent. (A) A549 cells were treated with $50 \mathrm{nM}$ leptomycin for increasing time until $36 \mathrm{~h}$ after which nuclear and cytoplasmic lysates were analysed by Western blotting using anti-p120 antibody pp120. Significant nuclear enrichment of p120ctn peaked by $18 \mathrm{~h}$. (B) We transiently transfected the p120-1A/3A and p120-NLS-1A/3A plasmids into A549 cells, then added $50 \mathrm{nM}$ LMB for $18 \mathrm{~h}$. Cytoplasmic and nuclear fractions of cells transfected with the vector alone (Empty), p120-1A/3A, p120-1A/3A+LMB and p120-NLS-1A/3A, were analysed by Western blotting to assess the relative levels of p120ctn and Kaiso protein within each compartment.

Kaiso was not affected by p120ctn-1A overexpression (Fig. 3B, compare lane 2 to lane 4 and lane 9 to lane 11), though LMB also partially inhibited nuclear export of p120ctn isoform 1 (Fig. 3B, compare lane 9 to lane 11).

Abnormal expression of p120ctn serine 288 phosphorylation in lung cancer tissue and cells. In comparison to the other serine/threonine (S/T) site, only S288 phosphorylation was detected in nucleus (26). We examined S288 phoshorylation in lung tumors. As shown in Fig. 4A, immunohistochemistry of normal bronchial epithelium and alveolar cells showed expression and localization of p120ctn primarily in the membrane. In contrast, S288 phoshorylation expression was almost absent. However, reduced or absent membrane expression with increased cytoplasmic expression was observed for p120ctn and S288 phoshorylation in lung squamous cell carcinoma and adenocarcinoma (Fig. 4A). Western blotting was used to evaluate $\mathrm{S} 288$ phoshorylation expression in 20 NSCLC and paired non-tumorous lung tissues of the same case. As shown in Fig. 4B, the significant higher level of S288 phosphorylation was found in NSCLC samples in comparison to the non-tumorous counterparts (Fig. $4 \mathrm{~B}, \mathrm{P}<0.01$ ). Based on S288 phosphorylation expression in lung tumors tissue, we examined three lung cancer cell lines and normal bronchial epithelium HBE, using nuclear-cytoplasmic extraction analysis to measure cellular distribution of S288 phosphorylation. As shown in Fig. 4C, the phospho-S288 signal was particularly striking in A549 and H460 cells when compared to HBE cells.
The serine 288 phosphorylation on p120ctn regulates redistribution of Kaiso. To determine whether S288 phosphorylation (Ps288) was needed for binding to Kaiso (Fig. 5A), immunoprecipitation assay was carried out and showed that the higher level Kaiso co-precipitated with p120ctn, using Ps288 as bait as opposed to pp120 (Fig. 5A, compare lane 2 to lane 3). The ability of Ps288 to recognize p120ctn immunoprecipitation was abrogated by dephosphorylation due to incubation with the $\lambda$ phosphatase reaction buffer (lane 4 ), indicating that S288 phosphorylation could be important for binding with Kaiso. However, the p120ctn could not co-precipitate with Kaiso using the Kasio antibody as bait (Fig. 5A, lanes 5 and 6). We then extracted the nuclear-cytoplasmic protein in different phosphorylated states induced by serum starvation, the PKC pathway activitor, PMA, and the PMA inhibitor, BisI. S288 site phosphorylation was strongly upregulated by serum starvation for $18 \mathrm{~h}$ in A549 cells (Fig. 5B), and these effects were readily reversed by serum add-back (Fig. 5D). The PKC activator, PMA, induced dramatic desphosphorylation at the S288 site in A549 cells after $30 \mathrm{~min}$ (Fig. 5C). These changes were completely blocked by the PKC inhibitor, BisI, which alone had no effect (Fig. 5E). As shown in Fig. 5F, the comparatively high level of Ps288 induced by serum starvation in the nucleus versus the cytoplasm, related to higher level Kaiso and coprecipitated with p120ctn in nucleus (Fig. 5F, compare lane 5 to lane 6). However, the dephosphorylation of S288 induced by PMA related to lower level Kaiso and co-precipitated with p120ctn in the nucleus (compare lane 5 to lane 7). These data 
A

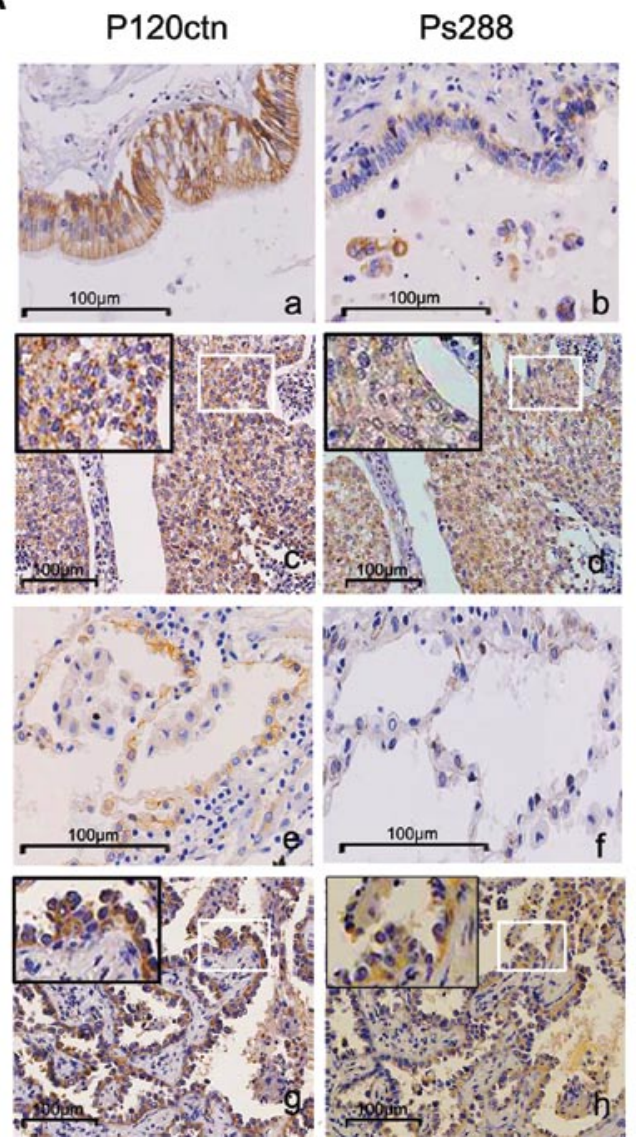

B
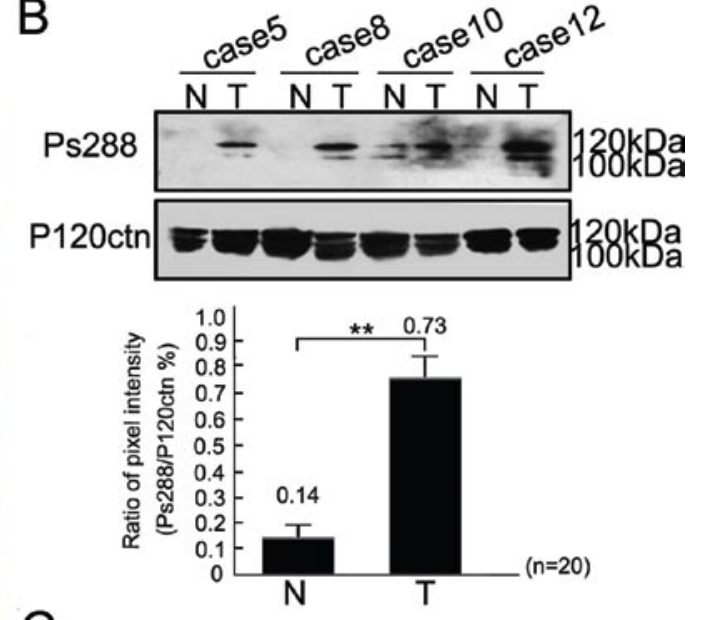

C

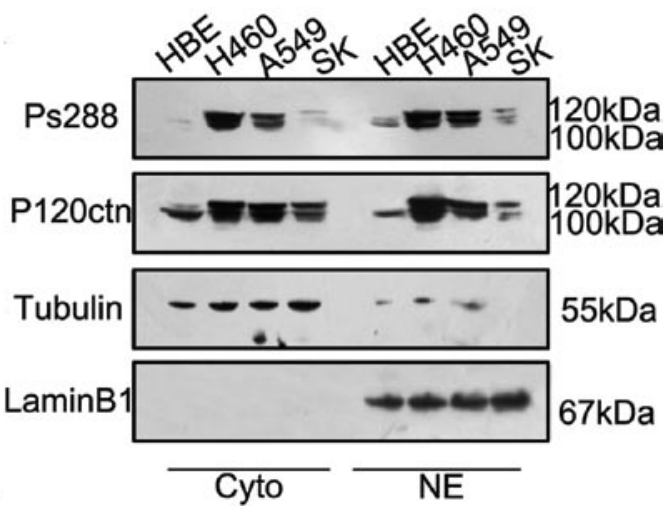

Figure 4. Abnormal expression of p120ctn serine 288 phosphorylation in lung cancer tissue and cells. (A) Immunohistochemical analysis of p120ctn and ps288 expression. (a-b) Normal bronchial epithelium, (c-d) squamous cell carcinoma, (e-f) alveolar cell, (g-h) adenocarcinoma. (B) Matched tumourous (T) and surrounding non-tumorous (N) tissues from 8 of 20 NSCLC patients were analysed by Western blotting. The ratio between the density of ps 288 and p120ctn of the same patient was calculated and expressed graphically. Data are expressed as the means $\pm \mathrm{SD}$. Columns, mean $(\mathrm{n}=20)$, ${ }^{* *} \mathrm{P}<0.01 .(\mathrm{C})$ Cytoplasmic and nuclear fractions of HBE, H460, A549 and SK cell lines were analysed by Western blotting to assess the relative levels of total and phospho-S288 p120ctn within each compartment.

suggest that the nuclear S288 phosphorylation can enhance binding with Kaiso.

To distinguish whether the S288 phosphorylation also regulated the subcellular redistribution of Kaiso, we overexpressed p120-1A/3A and treated with serum starvation, PMA and BisI transfected in A549 cell lines, in which p120ctn was knocked down by p120 siRNA. As shown in Fig. 6 , nuclear-cytoplasmic extraction did not show much high-level S288 phosphorylation induced by serum starvation in overexpressed p120-3A cells. Emerged cytoplasmic Kaiso protein was increased and nuclear Kaiso protein was decreased, compared to only p120-3A overexpression (Fig. 6, compare lane 3 to lane $5, \mathrm{P}<0.05$ and lane 14 to lane $16, \mathrm{P}<0.05)$. When we overexpressed p120-3A and added PMA, the dephosphorylation level of S288 was elevated, cytoplasmic Kaiso protein was decreased and nuclear Kaiso protein was increased, compared to only p120-3A overexpression (compare lane 3 to lane $7, \mathrm{P}<0.05$ and lane 14 to lane $18, \mathrm{P}<0.05)$. When we overexpressed p120-3A, then added PMA followed by serum starvation, a similar level of cytoplasmic and nuclear Kaiso protein was obtained, compared to only p120-3A overexpression (compare lane 3 to lane 9, P>0.05 and lane 14 to lane 20, $\mathrm{P}>0.05)$. These data demonstrate that the $\mathrm{S} 288$ phosphorylation in p120ctn isoform 3 may be related to the subcellular redistribution of Kaiso.

To futher characterize the phosphorylation sites involved in this regulatory step, we mutated Ser 288 to Ala. We then tranfected the mutant plasmids into A549 cell lines, in which p120ctn was knocked down. As shown in Fig. 7A, nuclearcytoplasmic extraction assay showed no nuclear-cytoplasmic redistribution of Kaiso when p120-3A(S288A) was overexpressed, as opposed to when p120-3A was overexpressed (Fig. 7A, compare lane 2 to lane 4 and lane 8 to lane 10). After overexpressing p120-3A(S288A), followed by serum starvation to induce the $\mathrm{S} / \mathrm{T}$ phosphorylation, no nuclear-cytoplasmic redistribution of Kaiso protein was observed (compare lane 2 to lane 6 and lane 8 to lane 12). These results indicate that the subcellular redistribution of Kaiso may be really regulated by serine 288 phosphorylation, but not by the other S/T sites in p120ctn. Similar results were also observed in A549 cell lines by the overexpression of mutated Ser 288 to Glu plasmids (Fig. 7B).

The serine and threonine phosphorylation in pl20ctn promotes lung cancer cell invasion. The ectopic overexpression of the p120ctn isoform $1 / 3$ has been associated with the formation of 
A

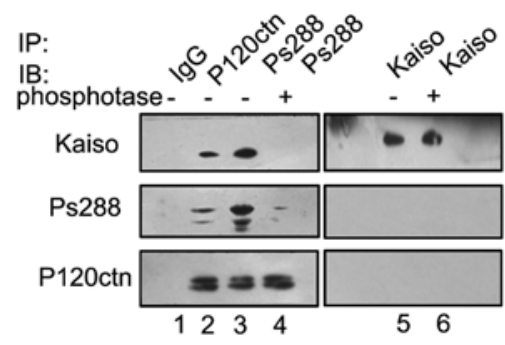

D

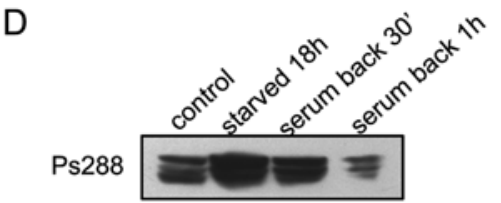

E

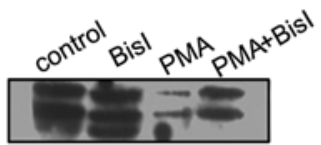

B
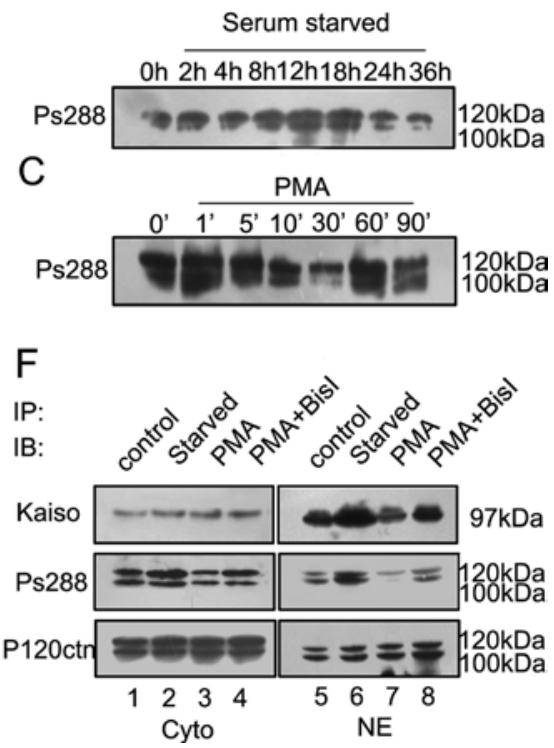

Figure 5. S288 phosphorylation and dephosphorylation. (A) Kaiso was immunoprecipitated from A549 cells by IgG, p120ctn and Kaiso antibodies as bait. The immunoprecipitated complex was also incubated with LAR phosphatase (New England BioLabs, Ipswich, MA 1800 U/ul) for 30 min before denaturation and electrophoresis. The membrane was probed with anti-Kaiso antibody. After removal of the anti-Kaiso antibody, it was reprobed with anti-p120 mAb pp120, Ps288. (B) A549 cells were treated with DMEM containing 0.1\% FBS for 2 to $36 \mathrm{~h}$ after which lysates were prepared and analysed by Western blotting with anti-Ps288 mAb. (C) A549 cells were treated with PMA (200 nM) for 1 to 90 min after which lysates were prepared and analysed by Western blotting with anti-Ps288 mAb. (D) The lysates from untreated A549 cells (control), the ones treated by serum starvation (serum-starved) for $18 \mathrm{~h}$, or serum starvation and then DMEM containing 10\% FBS for $30 \mathrm{~min}$ and $1 \mathrm{~h}$ (serum starved + serum back $30 \mathrm{~min} / 1 \mathrm{~h}$ ) were also analysed by Western blotting with anti-Ps $288 \mathrm{mAb}$. (E) The lysates from untreated A549 cells (control), the ones treated with PMA (200 nM) for $30 \mathrm{~min}$, BisI (1 $\mu \mathrm{M})$ for 10 min, and BisI after PMA treatment (PMA+BisI) were analysed by Western blotting with anti-Ps288 mAb. (F) A549 cells were serum-starved for $18 \mathrm{~h}$ (starved) or incubated with the PKC pathway activator PMA (200 nM) for $30 \mathrm{~min}$ (PMA). PMA was added for $30 \mathrm{~min}$ and PMA inhibitor BisI (1 $\mu \mathrm{M})$ for 10 min (PMA + BisI), then nuclear and cytoplasmic lysates were immunoprecipitated by Ps288 antibody and analysed by Western blotting with anti-Kaiso, Ps288 and pp120 antibodies.
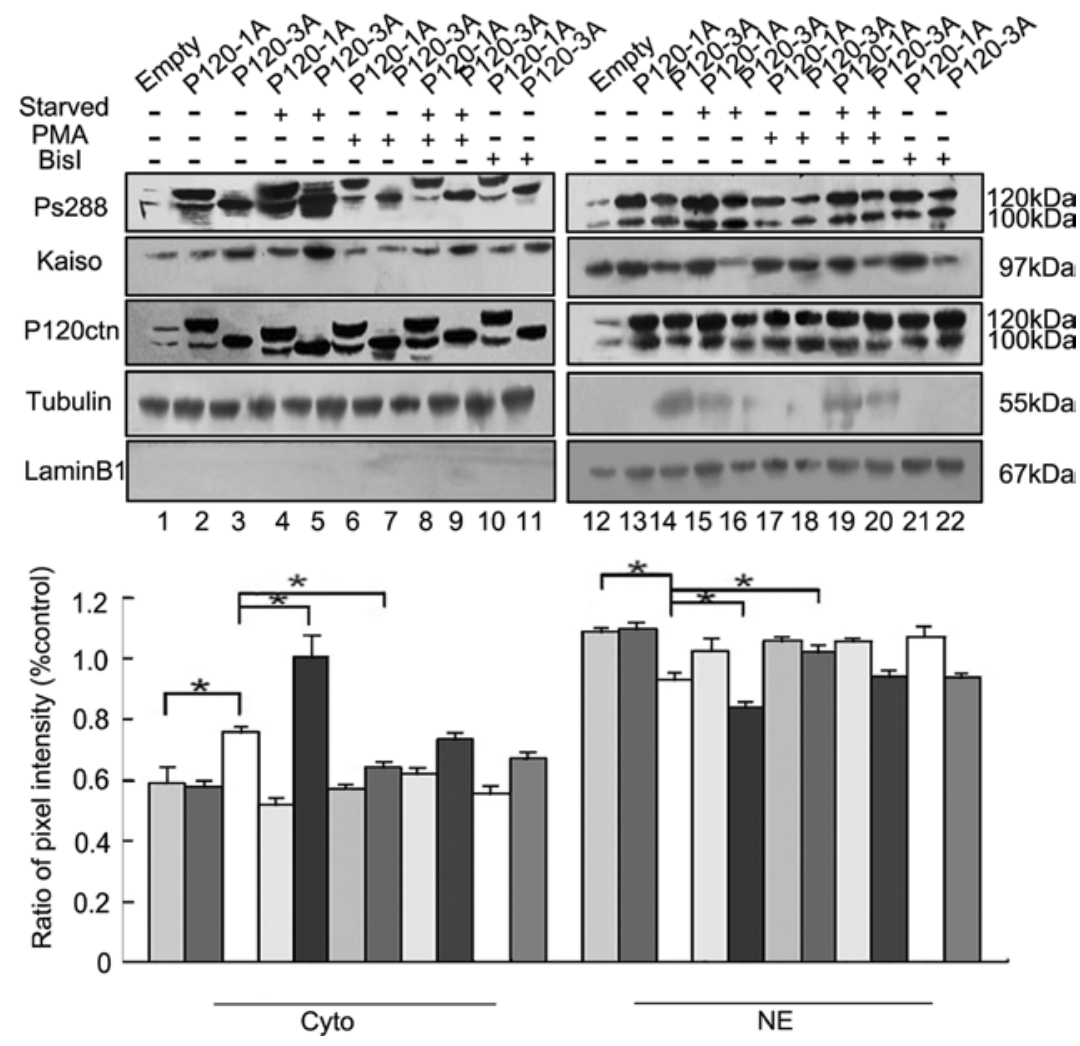

Figure 6. The serine 288 phosphorylation on p120ctn regulates the redistribution of Kaiso. Nuclear-cytoplasmic extraction was carried out in stably transfected p120 RNAi A549 cell lines, transiently transfected with the vector alone (Empty), or p120-1A/3A plasmids (p120-1A/3A) and which were then serum-starved, PMA, PMA+BisI, BisI treatment after post-transfection $24 \mathrm{~h}$. The lysates were analysed by Western blotting using anti-Kaiso, Ps288 and p120ctn antibodies. For the quatification of band density, the fold elevation of Kaiso versus lamin B1 or tubulin was displayed as the mean $\pm \mathrm{SD}$. "P<0.05. 


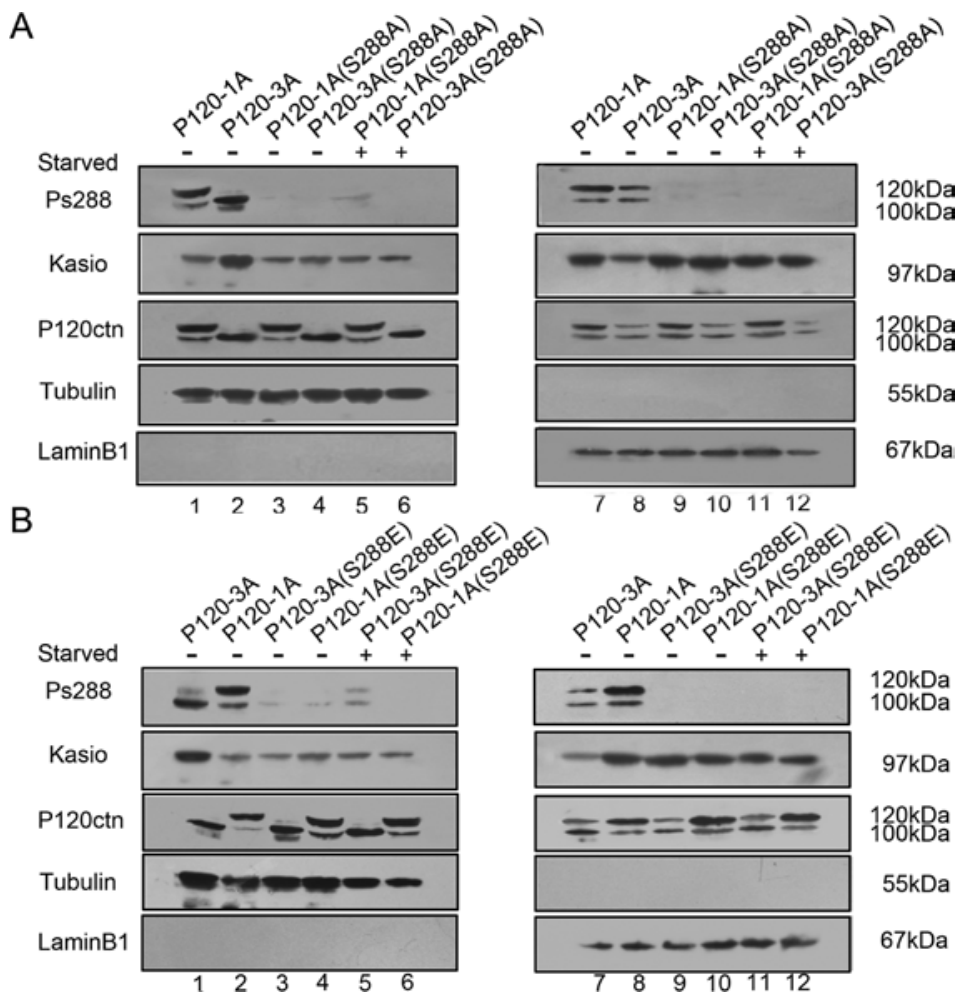

Figure 7. The relation between the serine 288 point mutant of p120ctn and the relocalization of Kaiso. (A) Nuclear-cytoplasmic extraction was carried out in stably transfected p120 RNAi A549 cell lines, transiently transfected with 120-1A/3A plasmids, or mutant plasmids P120-1A/3A(S288A) and which were then serum-starved. The lysates were then analysed by Western blotting using the above antibodies. (B) Cells transiently transfected with the mutant plasmids P120-1A/3A(S288E) were treated as above, and the lysates were analysed by Western blotting.

cellular extensions reminiscent of filopodia and lamellipodia to induce branching phenotype (28). As shown in Fig. 8A, we found a significant increase in the number of stress fiber marginalization, filopodia and membrane ruffles formation which was induced by the phosporylation of $\mathrm{S} / \mathrm{T}$ via serum starvation treatment. However, enhanced protrusion formation was relieved by PMA desphosphorylation. Moreover, adding BisI reversed the PMA effect to induce stress fiber marginalization, and the formation of filopodia and membrane ruffles as shown by F-actin stainning (Fig. 8A).

In order to investigate the effect on cell invasion with the emergance of a branching phenotype, we utilized an invasion assay to assess the ability of A549 and H460 cells to invade the Matrigel in different phosphorylated states of overexpressed p120-1A/3A. Enhanced invasive ability occurred in the presence of $\mathrm{S} / \mathrm{T}$ phosphorylation after serum starvation. Then the addition of PMA resulted in a marked reduction in invasive ability compared to treatmetn without PMA. Furthermore, the treatment with the PMA inhibitor, BisI, reversed the enhancement of invasive ability (Fig. 8B). However, the invasive ability was unchanged by treatment with p120-1A or p120-3A(S288E) and serum starvation compared to serum starvation alone (Fig. 8B). These data suggest that the phosphorylated state of p120ctn S/T sites and not the S288 sites can determine actin remodelling and promote cell invasion.

\section{Discussion}

Although nuclear and cytoplasmic Kaiso couple with p120ctn $(22,23)$, whether Kaiso shuttles out of the nucleus via p120ctn and via which p120ctn isoform, is yet to be determined. Relatively little knowledge is available regarding the phosphorylated p120ctn in the nucleus. In this study, we confirm that Kaiso shuttles out of the nucleus via p120ctn isoform 3 through the CRM-1 export pathway, but the nuclear import was independent of p120ctn. S/T phosphorylation in p120ctn enhanced binding with Kaiso and promoted the lung cancer cell invasion.

In this study, we indicate that only the p120ctn isoform 3 interacts with Kaiso. The results are in accordance with those by Daniels and Reynolds, that the very low levels of p120ctn isoform 1 in cells might preclude the detection of an interaction with Kaiso $(10,29)$. However, we demonstrate the inefficient immunoprecipitation of p120ctn mAb $6 \mathrm{H} 11$, which recognizes p120ctn isoform 1, in BE1 cell lines with high nuclear level of p120ctn isoform 1. We found that the lack of coiled coil domain on p120-1A, rescued the binding with Kaiso. Therefore, it is possible that the additional amino-terminal 46-101 amino acids in 120ctn isoform 1 block Kaiso binding by steric interference due to either modified p120 protein folding or recruitment of some other presently unknown p120-interacting protein. We also found that reciprocal co-immunoprecipitates of p120ctn with Kaiso mAb were unsuccessful. Possibly these $\mathrm{mAb}$ regions of Kaiso were sterically blocked by association with p120ctn.

NES is only encoded by exon B in p120ctn, and p120ctn also shuttles between the nucleus and cytoplasm via the other undefined sequences or signals $(19,30)$. We overexpressed p120-1A/3A to preclude the exon B-encoded NES, and then used LMB to inhibit the CRM-1 nuclear export pathway. This 
A

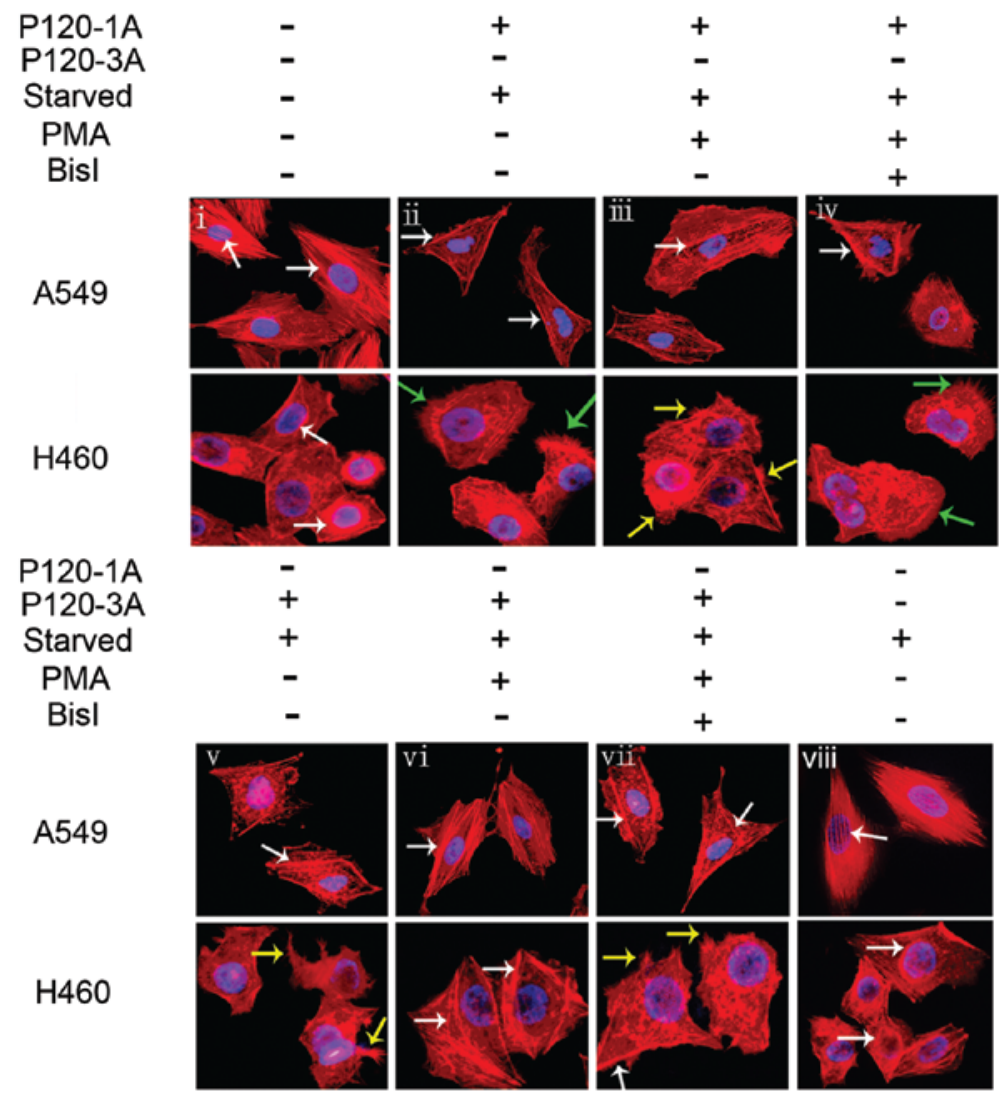

B
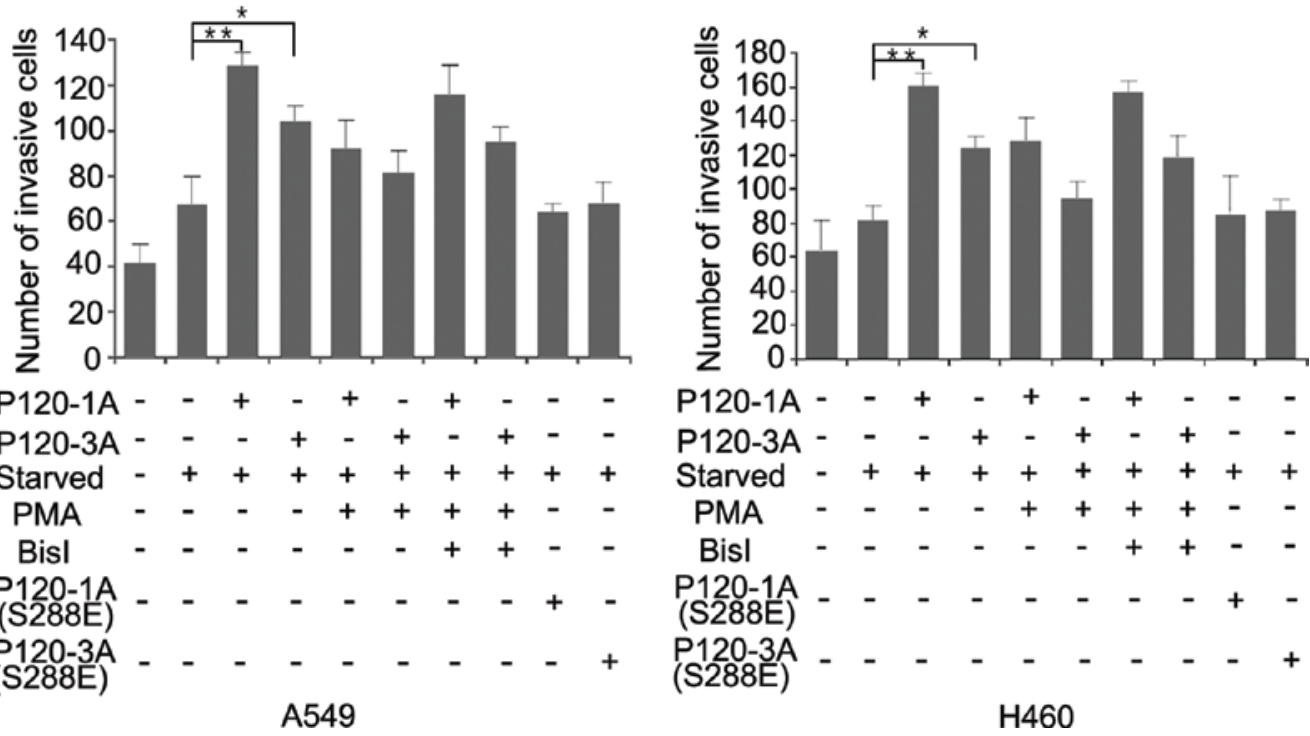

Figure 8. The serine and threonine phosphorylation in p120ctn promotes lung cancer cell invasion. (A) A549 cells and H460 cells were transiently transfected with the vector alone (i), p120-1A (ii-iv), p120-3A (v-vii). Post-transfection 24 h, they were serum-starved (starved; ii, v), and then PMA was added (starved+PMA; iii and vi), BisI was added (starved+PMA+BisI; iv, vii) or they were only starved (viii). F-actin was visualized by costaining with Alexa Fluor 546-labeled phalloidin (red) in A549 cells and H460 cells. The cells were treated as above. White arrows show stress fiber, yellow arrows show filopodia, green arrows show membranous ruffles. (B) A549 cells and H460 cells were transiently transfected with the vector alone, p120-1A, or p120-3A, and were then serum-starved; starved+PMA; starved+PMA+BisI. Transiently transfected mutant plasmids p120-1A(S288E), p120-3A(S288E) were serum-starved and then seeded on transwell filters and allowed to invade into Matrigel. The quantification of invasion is expressed as the mean $\pm \mathrm{SD}$. ${ }^{*} \mathrm{P}<0.05,{ }^{* *} \mathrm{P}<0.01$.

partially enriched nuclear p120ctn successfully. This demonstrates that there was undefined NES in p120ctn.

p120ctn is a multisite substrate for both tyrosine and $\mathrm{S} / \mathrm{T}$ kinases. The peculiar location of the phosphorylation sites at two distinct regions of p120ctn is two regulatory domains. All identified tyrosine phosphorylation sites are localized at the
$\mathrm{N}$-terminal end, where the majority of them cluster within a short stretch of amino acids together with several S/T phosphorylation sites. At the opposite end of p120ctn, the C-terminus harbours two phosphorylable $\mathrm{S} / \mathrm{T}$ residues (31). The tyrosine sites phosphorylated upon physiological activation of RTKs may only partially overlap with those phosphorylated 
directly by an aberrant tyrosine kinase ( $\mathrm{v}-\mathrm{Src}$ ), and can participate in the regulation of the formation and stabilization of cadherindependent adhesive contacts (32). As opposed to p120ctn tyrosine phosphorylation which is generally absent until transiently induced by various stimuli, unidentified kinases are responsible for the constitutive phosphorylation of $\mathrm{p} 120 \mathrm{ctn}$ at $\mathrm{S} / \mathrm{T}$ residues. However, the pharmacological activation of PKC selectively promotes phosphorylation of a specific serine at the C-terminus, and concomitantly induces the partial dephosphorylation of multiple sites at the N-terminus (33). While ligand-activated EGFR and PDGFR stimulates the S/T phosphorylation of $\mathrm{p} 120 \mathrm{ctn}$, the activation of VEGFR has been reported to induce $\mathrm{S} / \mathrm{T}$ deposphorylation at the $\mathrm{N}$-terminus $(34,35)$. Significant dephosphorylation of p120ctn has been also observed in response to inflammatory agents, such as histamine and thrombin, where PKC-dependent and-independent pathways modulate p120ctn S/T phosphorylation $(36,37)$. The phosphorylation of p120ctn in $\mathrm{S} / \mathrm{T}$ residues has also been implicated as a negative regulator of adhesion in colo-205 cells, where unknown consitutively active signalling pathways induce extensive phosphorylation of p120ctn (38). These observations lead to the speculation that $\mathrm{Y}$ and $\mathrm{S} / \mathrm{T}$ phosphorylation may actually influence each other and require a coordinate regulation. Multisite phosphorylation enables p120ctn to differentially respond to and interpret multiple incoming signals.

Currently, a number of $\mathrm{S} / \mathrm{T}$ site specific antibodies are available. We used Ps288 mAb, due to a previous report, that this site phosphorylation event may occur within the nuclear compartment (26). Studies have described that serum starvation can induce the S/T phosphorylation (S268, S288 and T310) in p120ctn and that most of the $\mathrm{S} / \mathrm{T}$ dephosphorylation is dependent on the PKC activator, PMA (26). We discovered that the S288 phosphorylation and dephosphorylation can also be induced by PMA. In a previous study, it was found that S288 phosphorylation was significantly phosphorylated in the absence of membrane localization, and occured in the nucleus, although the function of this event was not clear. In this study, we found that the S288 phosphorylation level was elevated in lung tumor tissue and cell lines, and that S288 phosphorylation enhanced the binding affinity with Kaiso, and promoted Kaiso shuttling out of the nucleus. We also found that actin remodelling was induced by p120ctn $\mathrm{S} / \mathrm{T}$ phosphorylation via serum starvation. Meanwhile, p120ctn S/T phosphorylation promoted cell invasion. We believe that this morphological change and biological behaviour are due to p120ctn phosphorylation, although the exact mechanism remains obscure. It coud be that p120ctn S288 phosphorylation relieves the Kaiso-mediated transcriptional repression of $M M P-7$ and MTA2. Another possibility is the p120ctn $\mathrm{S} / \mathrm{T}$ phosphorylation effect on cytoskeleton remodeling via modulation of Rho-family GTPase activity (7). Further experiments will be required to elucidate these possibilities.

In summary, our data suggest p120ctn isoform 3 assists Kaiso in shuttling out of the nucleus via S288 phosporylation, through the CRM-1 export pathway. Moreover, S/T phosphorylation in p120ctn promotes lung cancer cells invasion. To further understand the role of p120ctn isoforms in the nucleus and the effect of $\mathrm{S} / \mathrm{T}$ phosphorylation on the biological function of p120ctn, further studies are required.

\section{Acknowledgements}

We acknowledge the generosity of Dr A.B. Reynolds and Dr J.M. Daniel for the mp120-1A/3A and Kaiso plasmids. Thanks to Dr J. Zheng for providing BE1 and LH7 cell lines. This study was supported by grants from the National Natural Science Foundation of China (no. 30870977 to E.-H.W., no. 30901475 to Y.W., and no. 30900562 to Y.L.).

\section{References}

1. Davis MA, Ireton RC and Reynolds AB: A core function for p120ctn cateninin cadherin turnover. J Cell Biol 163: 525-534, 2003.

2. Peifer $\mathrm{M}$, Berg $\mathrm{S}$ and Reynolds $\mathrm{AB}$ : A repeating amino acid motif shared by proteins with diverse cellular roles. Cell 76: 789-791, 1994.

3. Daniel JM and Reynolds AB: The tyrosine kinase substrate p120cas binds directly to E-cadherin but not to the adenomatous polyposis coli protein or alpha-catenin. Mol Cell Biol 15: 4819-4824, 1995.

4. Thoreson MA, Anastasiadis PZ, Daniel JM, et al: Selective uncoupling of p120ctn from E-cadherin disrupts strong adhesion. J Cell Biol 148: 189-201, 2000.

5. Ohkubo T and Ozawa M: p120(ctn) binds to the membraneproximal region of the E-cadherin cytoplasmic domain and is involved in modulation of adhesion activity. J Biol Chem 274: 21409-21415, 1999.

6. Grosheva I, Shtutman M, Elbaum M and Bershadsky AD: p120 catenin affects cell motility via modulation of activity of Rho-family GTPases, a link between cell-cell contact formation and regulation of cell locomotion. J Cell Sci 114: 695-707, 2001.

7. Nobes CD and Hall A: Rho, rac and cdc42 GTPases: regulators of actin structures, cell adhesion and motility. Biochem Soc Trans 23: 456-459, 1995.

8. Anastasiadis PZ, Moon SY, Thoreson MA, et al: Inhibition of RhoA by p120 catenin. Nat Cell Biol 2: 637-644, 2000.

9. Liu Y, Wang Y, Zhang Y, et al: Abnormal expression of p120catenin, E-cadherin, and small GTPase is significantly associated with malignant phenotype of human lung cancer. Lung Cancer 63: 375-382, 2009.

10. Daniel JM and Reynolds AB: The catenin p120(ctn) interacts with Kaiso, a novel BTB/POZ domain zinc finger transcription factor. Mol Cell Biol 19: 3614-3623, 1999.

11. Van Hengel J, Vanhoenacker P, Staes K and van Roy F: Nuclear localization of the p120ctn Armadillo-like catenin is counteracted by a nuclear export signal and by E-cadherin expression. Proc Natl Acad Sci USA 96: 7980-7985, 1999.

12. Aho S, Levansuo L, Montonen O, Kari C, Rodeck U and Uitto J: Specific sequences in p120ctn determine subcellular distribution of its multiple isoforms involved in cellular adhesion of normal and malignant epithelial cells. J Cell Sci 115: 1392-1402, 2002.

13. Yanagisawa M, Huveldt D, Kreinest P, et al: A p120 catenin isoform switch affects Rho activity, induces tumor cell invasion and predicts metastatic diseases. J Biol Chem 283: 18344-18354, 2008.

14. Daniel JM, Spring CM, Crawford HC, Reynolds AB and Baig A: The p120(ctn)-binding partner Kaiso is a bi-modal DNA-binding protein that recognizes both a sequence-specific consensus and methylated CpG dinucleotides. Nucleic Acids Res 30: 2911-2919, 2002.

15. Prokhortchouk A, Hendrich B, Jorgensen H, et al: The p120 catenin partner Kaiso is a DNA methylation-dependent transcriptional repressor. Genes Dev 15: 1613-1618, 2001.

16. Park JI, Kim SW, Lyons JP, et al: Kaiso/p120-catenin and TCF/ beta-catenin complexes coordinately regulate canonical Wnt gene targets. Dev Cell 8: 843-854, 2005.

17. Spring CM, Kelly KF, O'Kelly I, Graham M, Crawford HC and Daniel JM: The catenin p120ctn inhibits Kaiso-mediated transcriptional repression of the beta-catenin/TCF target gene matrilysin. Exp Cell Res 305: 253-265, 2005.

18. Rodova M, Kelly KF, VanSaun M, Daniel JM and Werle MJ: Regulation of the rapsyn promoter by kaiso and delta-catenin at the neuromuscular junction. Mol Cell Biol 24: 7188-7196, 2004. 
19. Kelly KF, Spring CM, Otchere AA and Daniel JM: NLSdependent nuclear localization of p120ctn is necessary to relieve Kaiso-mediated transcriptional repression. J Cell Sci 117: 2675-2686, 2004.

20. Roczniak-Ferguson A and Reynolds AB: Regulation of p120catenin nucleocytoplasmic shuttling activity. J Cell Sci 116: 4201-4212, 2003.

21. Kelly KF, Otchere AA, Graham M and Daniel JM: Nuclear import of the BTB/POZ transcriptional regulator Kaiso. J Cell Sci 117: 6143-6152, 2004.

22. Dai SD, Wang Y, Jiang GY, et al: Kaiso is expressed in lung cancer: Its expression and localization is affected by p120ctn. Lung Cancer 67: 205-215, 2009.

23. Soubry A, van Hengel J, Parthoens E, et al: Expression and nuclear location of the transcriptional repressor Kaiso is regulated by the tumor microenvironment. Cancer Res 65: 2224-2233, 2005.

24. Castaño J, Solanas G, Casagolda D, et al: Specific phosphorylation of p120-Catenin regulatory domain differently modulates its binding to RhoA. Mol Cell Biol 27: 1745-1757, 2007.

25. Potter MD, Barbero S and Cheresh DA: Tyrosine phosphorylation of VE-cadherin prevents binding of p120ctn- and beta-catenin and maintains the cellular mesenchymal state. J Biol Chem 280: 31906-31912, 2005.

26. Xia X, Carnahan RH, Vaughan MH, Wildenberg GA and Reynolds AB: p120 serine and threonine phosphorylation is controlled by multiple ligand-receptor pathways but not cadherin ligation. Exp Cell Res 312: 3336-3348, 2006.

27. Travis WD, Brambilla E, Muller-Hermelink HK and Harris CC: Pathology and genetics of tumours of the lung, pleura, thymus and heart. In: World Health Organization Classification of Tumours. IARC Press, Lyon, 2004.

28. Reynolds AB, Daniel JM, Mo YY, Wu J and Zhang Z: The novel catenin p120cas binds classical cadherins and induces an unusual morphological phenotype in NIH3T3 fibroblasts. Exp Cell Res 225: 328-337, 1996.
29. Daniel JM: Dancing in and out of nucleus: p120(ctn) and the transcriptional factor Kaiso. Biochim Biophys Acta 1773: 59-68, 2007.

30. Bogerd HP, Fridell RA, Benson RE, Hua J and Cullen BR: Protein sequence requirements for function of the human T-cell leukemia virus type 1 Rex nuclear export signal delineated by a novel in vivo randomization-selection assay. Mol Cell Biol 16: 4207-4214, 1996.

31. Reynolds $A B$ and Roczniak-Ferguson A: Emerging roles for p120-catenin in cell adhesion and cancer. Oncogene 23: 7947-7956, 2004.

32. Mariner DJ, Anastasiadis P, Keilhack H, Bohmer FD, Wang J and Reynolds AB: Identification of Src phosphorylation sites in the catenin p120ctn. J Biol Chem 276: 28006-28013, 2001.

33. Xia X, Mariner DJ and Reynolds AB: Adhesion-associated and PKC modulated changes in serine/threonine phosphorylation of p120ctn catenin. Biochemistry 42: 9195-9204, 2003.

34. Downing JR and Reynolds AB: PDGF, CSF-1, and EGF induce tyrosine phosphorylation of p120ctn, a pp60src transformationassociated substrate. Oncogene 6: 607-613, 1991.

35. Wong EY, Morgan L, Smales C, Lang P, Gubby SE and Staddon JM: Vascular endothelial growth factor stimulates dephosphorylation of the catenins p120 and p100 in endothelial cells. Biochem J 346: 209-216, 2000.

36. Ratclife MJ, Smales C and Staddon JM: Dephosphorylation of the catenins p120 and p100 in endothelial cells in response to inflammatory stimuli. Biochem J 338: 471-478, 1999.

37. Konstantoulaki M, Kouklis P and Malik AB: Protein kinase $\mathrm{C}$ modifications of VE-cadherin, p120, and beta-catenin contribute to endothelial barrier dysregulation induced by thrombin. Am J Physiol Lung Cell Mol Physiol 285: L434-L442, 2003.

38. Aono S, Nakagawa S, Reynolds AB and Takeichi M: p120(ctn) acts as an inhibitory regulator of cadherin function in colon carcinoma cells. J Cell Biol 145: 551-562, 1999. 